\title{
Seguimiento microbiológico: estrategia para el mejoramiento del registro de la información en los procesos microbiológicos
}

\author{
Jaime Alberto López, Mónica Cecilia Cuartas, Olga Lucía Molina, \\ Ana Cristina Restrepo, Claudia Yarely Maya, Sergio Jaramillo \\ Laboratorio de Microbiología, Hospital Pablo Tobón Uribe, Medellín, Colombia.
}

El conocimiento sobre el qué, el quién, el cuándo, el dónde, el cómo y el porqué de cada proceso, la aplicación de un sistema de control de calidad y la retroalimentación de la evaluación permiten que la información sea analizada y empleada eficientemente en el mejoramiento continuo. Los objetivos del trabajo fueron: mejorar el registro de la información en los procesos microbiológicos, evaluar los resultados obtenidos con las estrategias empleadas, establecer una base de datos para proyectos de investigación y proponer un modelo de evaluación para estudios comparativos. Para esto, se realizó un estudio descriptivo prospectivo, y se utilizaron los datos de los cultivos microbiológicos solicitados a pacientes hospitalizados entre junio de 1997 y junio de 2003. Se diseñó un formato con las variables por analizar. Las fuentes de información fueron las solicitudes de laboratorio, los registros del laboratorio de microbiología y las historias clínicas. Se utilizó el programa Epilnfo 6 para analizar la información de 46.072 cultivos. La primera cifra corresponde al porcentaje en junio de 1997 y la segunda, a la de junio de 2003: persona que solicitó el cultivo (11\%-99\%), hora de la solicitud (9\%-85\%), solicitud completamente diligenciada (10\%-68\%), persona que recolectó la muestra $(0 \%-83 \%)$, hora de recolección de la muestra $(0 \%-77 \%)$ y, finalmente, hoja de trabajo microbiológica completa (78\%-96\%). El primer paso para el mejoramiento de cualquier proceso consiste en obtener y registrar la información pertinente y, para ello, la evaluación constante y la retroalimentación son fundamentales para el logro de este objetivo.

Palabras clave: registros, registros computarizados de pacientes, informática clínica, formatos, recolección de datos, bases de datos.

Development of a record-keeping strategy for improvement of information retention in microbiological processing

The improvement of microbiological information processing in clinical laboratories depends on retention of information concerning who, what, when, how, and why each process was performed, the implementation of quality control procedures, and finally, its evaluation. The four objectives to be addressed are as follows: (1) to improve the collection of information concerned with microbiological processes, (2) to evaluate results of implemented strategies, (3) to offer a model data base to be used in research projects, and (4) to propose an evaluation model for comparative studies. To do this, microbiological cultures were collected from hospitalized patients from June 1997 to June 2003. Data for the analytical matrix were obtained from lab requests, medical history and the microbiological data. Statistical analyses were performed in Epi-Info 6. The laboratory records for 46,072 microbiological cultures were analyzed. Completion levels in data collection were compared between years 1997 and 2003. Samples from 1997 and 2003 showed $11 \%$ and $99 \%$ of the request forms specifically requesting microbiological culture, $11 \%$ and $99 \%$ were completed in 1997 and 2003, respectively. For the same years, 9\% and $85 \%$ specifically stated the time of the request. Ten percent and $68 \%$, respectively, provided complete information. Zero and $83 \%$ respectively stated who had collected the sample. Zero and $77 \%$, respectively, specified the time of sample collection. Forms containing all relevent microbiological data were most complete with $78 \%$ and $96 \%$, respectively. A database with 44 
variables related to microbiological processes was created. In conclusion, improvement of microbiological data processing depends not only on the method of collection and completion of recorded information, but also on constant quality control and evaluation.

Key words: medical records, databases, clinical informatics, data formats, data collection.

El objetivo de las actividades realizadas en el laboratorio de microbiología debe ser el proporcionar un resultado oportuno y confiable que le permita al médico tomar la mejor decisión para su paciente. El mejoramiento continuo de la calidad de un sistema requiere del conocimiento sobre el qué, el quién, el cuándo, el dónde, el cómo y el porqué de cada uno de los procesos que lo componen. Además, la aplicación de un programa de control de calidad y la gestión de la rutina por medio del ciclo planear, hacer, verificar y actuar correctiva y preventivamente (PHVA) permiten el logro del objetivo inicialmente propuesto (1-3).

Las metas para la gerencia de los pasos de un proceso sólo pueden ser definidas después del conocimiento de su capacidad o evaluación del mismo, y de acuerdo con las expectativas del cliente del servicio $(1,2)$. Los ítems de control, que son las características medibles del servicio y cuya verificación debe garantizar la satisfacción del usuario, deben determinarse a través del proceso del desdoblamiento de la calidad (1).

El registro y el procesamiento de la información de las actividades realizadas en cada uno de los procesos permiten que ésta sea utilizada para retroalimentar el sistema, tomar decisiones de tipo administrativo y técnico, y verificar el cumplimiento de las metas propuestas.

La obtención de los datos que conforman la información son indispensables en las etapas de planeación, ejecución y verificación del ciclo PHVA. La evaluación periódica de la manera como son diligenciados los registros que contienen la información, permite que este proceso mejore y se mantenga constante en el tiempo.

Correspondencia:

Jaime Alberto López, Calle 78B No.69-240, Medellín, Colombia. Teléfono: 445 9286; fax: 4417955.

jlopez@ hptu.org.co.

Recibido: 29/01/04; aceptado: 26/07/04
En el Hospital Pablo Tobón Uribe (HPTU) de Medellín, como parte del mejoramiento continuo de la calidad, y para dar cumplimiento al decreto 77 de 1997 del Ministerio de Salud de Colombia, por el cual se reglamentan los requisitos y condiciones técnico-sanitarias de los laboratorios clínicos, específicamente en la parte de gestión y registro y documentación del laboratorio clínico (4), se decidió mejorar el registro de la información de los procesos relacionados con el Laboratorio de Microbiología, empleando las herramientas de la gerencia de la rutina (2). Además, estas actividades hacen parte de los requisitos para obtener la certificación bajo la norma ISO-90012000 (5), la cual fue obtenida por el Laboratorio de Microbiología del HPTU en marzo de 2004.

\section{Materiales y métodos}

\section{Tipo de estudio y población}

El presente estudio es de tipo descriptivo prospectivo. Se analizaron los registros de los cultivos microbiológicos solicitados a los pacientes hospitalizados en el HPTU entre junio de 1997 y junio de 2003.

EI HPTU es una entidad de cuarto nivel, privada, sin ánimo de lucro, fundada en 1970, y en la actualidad cuenta con 224 camas, 18 de ellas ubicadas en la unidad de cuidados intensivos, y que atiende todas las especialidades médicas, con excepción de psiquiatría y obstetricia. EI Laboratorio de Microbiología presta sus servicios tanto a los pacientes ambulatorios como a aquéllos que son hospitalizados en la institución.

\section{Registros y recolección de la información}

Se diseñó el formato "seguimiento microbiológico", el cual contiene, entre otras, las variables por analizar, considerando las diferentes etapas y procesos microbiológicos. El médico microbiólogo diligenció un formato por cada cultivo solicitado a un paciente que estuviera o que hubiera estado hospitalizado. 
Se estableció que por cada tipo de muestra remitida al laboratorio, se debería diligenciar un formato de "solicitud de laboratorio", diseñado con tal propósito y que, a la vez, se utilizaría como "hoja de trabajo" para registrar los procesos realizados con la muestra remitida, y los resultados obtenidos en el Laboratorio de Microbiología.

\section{Criterios de aceptabilidad}

Se calificó como aceptable el dato de "quién solicitó el cultivo" si la solicitud tenía el nombre legible del médico, vinculado o no con el hospital.

Se evaluó como "solicitud con los datos completos", si el formato de solicitud contenía el nombre del médico vinculado o no con el hospital, la fecha y hora de solicitud del cultivo, el tipo de muestra remitida, el origen de la muestra, el método de recolección de la misma (en el caso de tratarse de una muestra de orina), la impresión diagnóstica por la cual se solicitó el cultivo y la información de los antibióticos que estaba recibiendo e iban a iniciarse inmediatamente después de recolectada la muestra.

La "hoja de trabajo del Laboratorio de Microbiología" se evaluó como completa si contenía la siguiente información: fecha, hora y nombre de la bacterióloga responsable de realizar el cultivo, la evaluación de la calidad de la muestra remitida y el medio de transporte. En el caso de líquidos, secreciones, pus o tejidos, el recipiente en el que llegó la muestra. Con respecto a la coloración de Gram, se tuvo en cuenta la evaluación de la calidad del extendido o la muestra para realizarla, el resultado, la hora de la interpretación, la hora del reporte y el nombre de la bacterióloga responsable.

\section{Acciones de mejoramiento}

Las estrategias empleadas para mejorar el registro de la información fueron:

- Remitir y suministrar el instructivo "Solicitud de exámenes de laboratorio - Laboratorio de Microbiología" a los médicos y a todos los servicios del hospital.

- Realizar la inducción y entregar la circular del formato "Inducción - Laboratorio de
Microbiología" a los nuevos médicos vinculados.

Entregar para su lectura el instructivo del formato "Hoja de trabajo - Laboratorio de Microbiología" a todos las bacteriólogas.

- Realizar el proceso de inducción a cada nuevo bacterióloga.

- Entregar a las enfermeras la circular "Inducción - Laboratorio de Microbiología - Enfermería" y realizar una reunión de inducción en el momento de su vinculación con el hospital.

- Se informó telefónicamente y se remitió un comunicado a cada enfermera o auxiliar de enfermería que no habían anotado su nombre, la fecha y hora de recolección de la muestra.

Realizar el proceso de inducción a las auxiliares de laboratorio.

- Realizar el proceso de inducción a las secretarias del laboratorio.

- Evaluar bimensual o trimestralmente las solicitudes remitidas y remitir a los médicos que más solicitudes diligenciaron, el resultado de su evaluación.

- Evaluar bimensual o trimestralmente las hojas de trabajo del Laboratorio de Microbiología, y entregar a cada bacterióloga su evaluación.

- Evaluar bimensual o trimestralmente el registro de los datos de quién y cuándo recolectó las muestras, y remitir el informe a la jefa del Departamento de Enfermería, a las enfermeras coordinadoras de las áreas y a cada servicio del hospital.

- Evaluar bimensual o trimestralmente el proceso de recolección de las muestras de los hemocultivos por parte de las auxiliares de laboratorio y el personal de enfermería, y entregarles su evaluación correspondiente.

- Evaluar bimensual o trimestralmente el proceso de registro de las muestras para cultivo en el laboratorio, y entregar a las secretarias su evaluación correspondiente.

La información se registró y analizó empleando el programa Epilnfo 6, y para la elaboración de las 
gráficas se utilizó el programa Windows Excel 2000.

\section{Resultados}

Se recolectó y analizó la información correspondiente a 46.072 cultivos microbiológicos. El porcentaje de las "solicitudes de laboratorio" entregadas al Laboratorio de Microbiología, que en junio de 1997 era del 31\%, fue incrementándose hasta alcanzar el $100 \%$ en octubre de 1998 , y se sostuvo hasta junio de 2003. El dato del "nombre del médico que solicitó el cultivo", inicialmente del $15 \%$, pasó en diciembre de 1997 al 38\%, y al mes siguiente fue del $70 \%$. A partir de este momento, el porcentaje se fue incrementando hasta alcanzar el $100 \%$ en febrero de 1999, cifra que se ha mantenido casi constante desde entonces (figura 1).

El dato de la "hora en la cual fue solicitado el cultivo" presentó un comportamiento similar al dato de quién solicitó el cultivo, aunque las cifras fueron inferiores; inició con $11 \%$ y durante el seguimiento alcanzó cifras hasta del $92 \%$ y para junio de 2003 , el $85 \%$ de las solicitudes tenía el dato (figura 1).

En junio de 1997, las "solicitudes completamente diligenciadas" representaban el 10\%; en diciembre de 1999 alcanzó el máximo de $78 \%$ y, luego, descendió hasta alcanzar el $68 \%$ en junio de 2003 (figura 1).

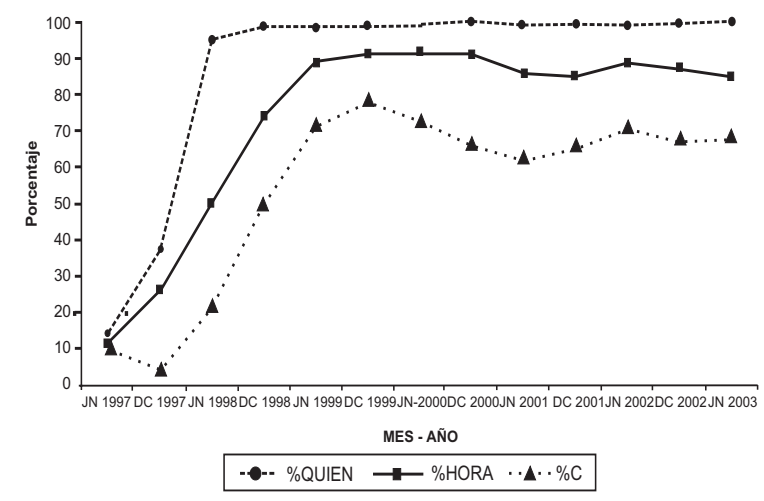

Figura 1. Porcentajes del dato del médico, la hora de solicitud, las solicitudes completamente diligenciadas de los cultivos de los pacientes hospitalizados, HPTU, junio/1997-junio/2003.

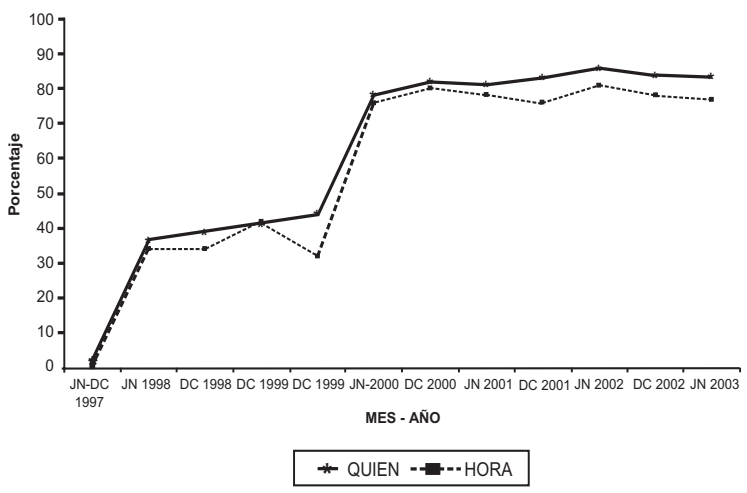

Figura 2. Porcentaje del dato de quién y cuándo recolectó la muestra de los cultivos de los pacientes hospitalizados, HPTU, junio/1997-junio/2003.

Al inicio del seguimiento, el nombre de la persona que había recolectado la muestra para el cultivo y la hora cuando se había realizado este procedimiento era desconocida en todos los casos; en 1998 y 1999, las cifras estuvieron entre el $35 \%$ y el $45 \%$; no obstante, en febrero y marzo de 2000 el porcentaje ascendió hasta el $65 \%$ en cuanto al nombre de la persona, y al $60 \%$ para la hora de la recolección de la muestra. En junio de 2003 estos niveles estaban en $83 \%$ y $77 \%$, respectivamente (figura 2 ).

Las "hojas de trabajo del Laboratorio de Microbiología" que se diligencian a todos los cultivos para aerobios, anaerobios, coprocultivos, urocultivos y hemocultivos positivos mostraron en junio de 1997 que el 78\% contenía toda la información requerida. Se observó un descenso en tal cifra en noviembre y diciembre del mismo año, para alcanzar un nivel superior al $90 \%$ en junio de 1998; en junio de 2003 el nivel era del $96 \%$.

Se estableció una base de datos con 44 variables relacionadas con los procesos microbiológicos, basados en la información registrada en los formatos descritos.

\section{Discusión}

En el presente estudio se exponen los resultados obtenidos durante el proceso de mejoramiento del registro de la información, de acuerdo con los procedimientos realizados en 46.072 cultivos microbiológicos durante 6 años de seguimiento 
prospectivo. Desconocemos la existencia de seguimientos similares a nivel nacional o internacional.

El propósito general del programa que hemos denominado "seguimiento microbiológico", es mejorar los procesos realizados en el Laboratorio de Microbiología por medio del sistema PHVA (2), que consiste, básicamente, en: planear, proponiendo las metas y definiendo los métodos y estrategias para alcanzarlas; hacer, educando y capacitando a las personas para la ejecución de las tareas, y recolectar la información para lograr verificar los resultados de las acciones realizadas para, posteriormente, actuar correctivamente y reiniciar de nuevo el ciclo (1-3).

Inicialmente, se establecieron los diferentes procesos que componían el ciclo de servicio del Laboratorio de Microbiología y, con base en ellos, se diseñó un formato que permitiera recolectar la información contenida en los diferentes registros. Destacamos que toda la información recolectada procede directamente del trabajo diario y se pretende continuar con este seguimiento, haciendo parte del trabajo cotidiano. Es por ello que cualquier laboratorio de microbiología puede y está en el deber de establecer procesos similares por las razones antes citadas, y porque se requiere únicamente del análisis de los registros que todo laboratorio debe poseer. Además, serviría de punto de referencia y comparación entre los diferentes centros de atención en salud para establecer estándares de calidad.

Uno de los objetivos del programa "seguimiento microbiológico" fue mejorar el registro de la información en los procesos microbiológicos, para lo cual se desarrollaron diferentes estrategias; infortunadamente, no es posible con los datos disponibles comparar las diferentes estrategias utilizadas, ya que no era uno de nuestros objetivos iniciales del estudio.

De los resultados obtenidos podemos exponer las siguientes observaciones: se logró que el 100\% de las solicitudes fueran entregadas al Laboratorio de Microbiología, el cual expidió la norma que exigía el diligenciamiento de un formato individual por cada tipo de muestra. La inducción del personal médico y paramédico, con respecto a la exigencia de escribir el nombre del médico solicitante y la posterior evaluación, representó un incremento significativo, de 38\% en diciembre de 1997 a $70 \%$ en enero de 1998. La estrategia de remitir a los médicos que solicitaban los cultivos, las evaluaciones sobre cómo estaban diligenciando las solicitudes, puede explicar el incremento en el porcentaje de las solicitudes completamente diligenciadas a partir de abril de 1998. Este parámetro alcanzó una meseta que no se ha logrado superar, a pesar de las múltiples estrategias emprendidas: la retroalimentación de las evaluaciones a los jefes de los departamentos médicos, la evaluación y retroalimentación personificada, la comunicación verbal y directa con algunos de los médicos, y el preguntar a los médicos con los porcentajes más bajos, las causas de su falta de cumplimiento.

Una vez más, se presencia el incumplimiento de las normas por parte de algunos médicos, como sucede con otros procedimientos más ampliamente estudiados como lo son, por ejemplo, el lavado de las manos, así como de otros estándares y manuales de procedimientos (6-8). En nuestro caso, hemos empleado dos de las estrategias descritas y empleadas con éxito relativo: la educación y la retroalimentación de los resultados (7). No hemos implementado hasta el momento estrategias de tipo administrativo como sería, por ejemplo, el no recibir las solicitudes sin todos los datos exigidos; aunque en un futuro, con la implementación de la historia clínica electrónica en el HPTU, se impondrán medidas de este tipo, debido a que este sistema de información exige el diligenciamiento completo de la solicitud de los exámenes, pero se presentarán posiblemente problemas en la digitación de las solicitudes como está descrito por otros autores (9).

De la calidad de la muestra depende la validez del resultado microbiológico (10) y, por tanto, es importante saber el quién, el cuándo y el cómo se realiza la recolección de ésta, información fundamental para dirigir las acciones de mejoramiento de manera personificada. Para mejorar en este aspecto, en 1998 y 1999 se empleó la estrategia de evaluación del proceso de recolección de las muestras por parte de enfermería y, para ello, se remitía el informe a las 
jefas de este personal por áreas, y se exponían los resultados en las carteleras de los servicios de hospitalización y urgencias; pero no produjo los resultados esperados y, por lo tanto, se inició en enero de 2000, la comunicación personal vía telefónica y por escrito con las personas responsables del proceso, invitándolas a anotar en la solicitud, su nombre, la fecha y la hora de la recolección. Esta nueva estrategia produjo un aumento de $45 \%$ a $82 \%$, al año de implementada.

Cuando observamos los resultados del registro que depende de las bacteriólogas, fue posible detectar el mismo fenómeno. Se presentó un descenso acentuado en las cifras en los meses finales de 1997 y a comienzos de 1998 que coincidió con un cambio en el formato de la hoja de trabajo; se implementó, entonces, una evaluación personificada en los primeros meses de 1998, que explicaría el ascenso y el mantenimiento de los porcentajes a partir de los primeros meses de este año. Este resultado fue similar a lo sucedido con los médicos tratantes.

Con la información obtenida y conservada es posible proponer nuevas investigaciones y, en cualquier momento, agregar nuevas variables y consultar los datos en las historias clínicas. Otra posibilidad que se abre es realizar estudios con los laboratorios de microbiología de otros hospitales con el propósito de comparar los resultados y establecer estándares de calidad.

En conclusión, por las observaciones derivadas de nuestro seguimiento, el mejoramiento en el registro de la información, que es básica para la el seguimiento del sistema microbiológico, puede lograrse con base en actividades de educación y evaluación, realizadas de manera personificada y constante, y retroalimentando a las personas involucradas en los procesos y, obviamente, con la voluntad de las personas responsables de la gestión de los procesos incluidos en el ciclo de servicios de los laboratorios de microbiología.

\section{Referencias}

1. Helman H. Gerencia de la rutina. Gerencia del día a día. Medellín: Centro de Gestión Hospitalaria-Fundación Chistiano Otonni; 1993.

2. Falconi V. Gerenciamiento de la rutina del trabajo cotidiano. Belo Horizonte: Universidad Federal de Minas Gerais, Fundación Chistiano Ottoni; 1992.

3. Soin SS. El ciclo de mejoramiento. En: Soin SS. Control de calidad total. México: Publi-Mex; 1997. p.91-123.

4. Colombia. Ministerio de Salud. Decreto número 77 de 1997. Diario Oficial. Año CXXXII. N. 42965. 23, Enero, 1997. p. 16.

5. ICONTEC. Norma técnica colombiana NTC-ISO9001. Sistemas de gestión de la calidad. Segunda actualización, Bogotá: Icontec; 2001.

6. Albert RK, Condie F. Hand-washing patterns in medical intensive-care units. N Engl J Med 1981;304:1465-6.

7. Greco PJ, Eisenberg JM. Changing physicians' practices. N Engl J Med 1993;329:1271-4.

8. Cabana MD, Rand CS, Powe NR et al. Why don't physicians follow clinical practice guidelines? A framework for improvement. JAMA 1999;282:1458-65.

9. Valenstein P, Meier F. Outpatient order accuracy. A College of American Pathologists Q-probes study of requisition order entry accuracy in 660 institutions. Arch Pathol Lab Med 1999;123:1145-50.

10. Miller JM, Holmes HT. Specimen collection, transport, and storage. En: Murray PR, Baron EJ, Pfaller MA, Tenover FC, Yolken RH, editors. Manual of clinical microbiology. $7^{\text {th }}$ ed. Washington, D.C.: ASM Press; 1999. p.33-63. 